\title{
Mediastinal tuberculous lymphadenitis presenting as superior vena cava syndrome
}

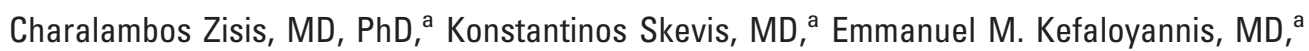

Konstantinos Avgoustou, MD, ${ }^{\mathrm{b}}$ and Ion Bellenis, MD, PhD, FETCS, ${ }^{a}$ Athens, Greece

$\mathrm{T}$

uberculous lymphadenitis without pulmonary manifestation is an uncommon entity in developed countries, and the possibility of tuberculous infection is usually ignored in the differential diagnosis of lymphadenopathy. Therefore appropriate treatment might be delayed. In many cases tuberculosis presents as fever of unknown origin but rarely with symptoms characteristic of superior vena cava obstruction, such as facial swelling, dyspnea, and cyanosis (SVCS).

Before the era of antibiotics, the vast majority of cases of SVCS $^{1,2}$ were due to infectious diseases, such as syphilis or tuberculosis, but nowadays, cancer is the main cause of this syndrome. ${ }^{3}$ In recent years, the outbreak of AIDS has led to an increased incidence of tuberculosis and its complications.

\section{Clinical Summary}

A 72-year-old male smoker (50 pack-years) was admitted to the hospital with a 4-week history of fever, malaise, and anemia. He presented with clinical signs of superior vena cava syndrome, whereas chest radiography revealed a mediastinal enlargement particularly caused by the right paratracheal space, where pathologic lymph nodes were suspected (Figure 1). Routine laboratory tests indicated anemia with normal white blood cell and platelet counts and an increased erythrocyte sedimentation rate. Tests for Leishmania species, Rickettsia species, Coxciella burnettii, Toxoplasma species, Bartonella species, and Widal-Wright were negative. Mantoux test results were positive, but the results of urine analysis were negative for acid-fast bacilli. Computed tomographic (CT) scanning of the chest depicted protracheal and paratracheal abnormal tissue with signs of compression over superior vena cava in the anterosuperior and middle mediastinal compartment (Figure 2). Those CT scan findings were characteristic for lymphoma or small cell lung carcinoma. The patient underwent videomediastinoscopy to establish a diagnosis in view of previous diagnostic problems. At the operation, enlarged paratracheal lymph node dissection was undertaken, and the histologic examination set the diagnosis of tuberculous adenopathy.

From the Department of Thoracic Surgery, Evangelismos General Hospital, ${ }^{\mathrm{a}}$ and the Department of Surgery, "Aghia Olga" General Hospital, ${ }^{\mathrm{b}}$ Athens, Greece.

Received for publication Jan 11, 2006; accepted for publication Feb 6, 2006.

Address for reprints: Charalambos Zisis, MD, 17A Patriarchou Grigoriou str, 166 74-Glyfada, Greece (E-mail: chzisis@otenet.gr).

J Thorac Cardiovasc Surg 2006;131:e11-2

$0022-5223 / \$ 32.00$

Copyright () 2006 by The American Association for Thoracic Surgery doi:10.1016/j.jtcvs.2006.02.003

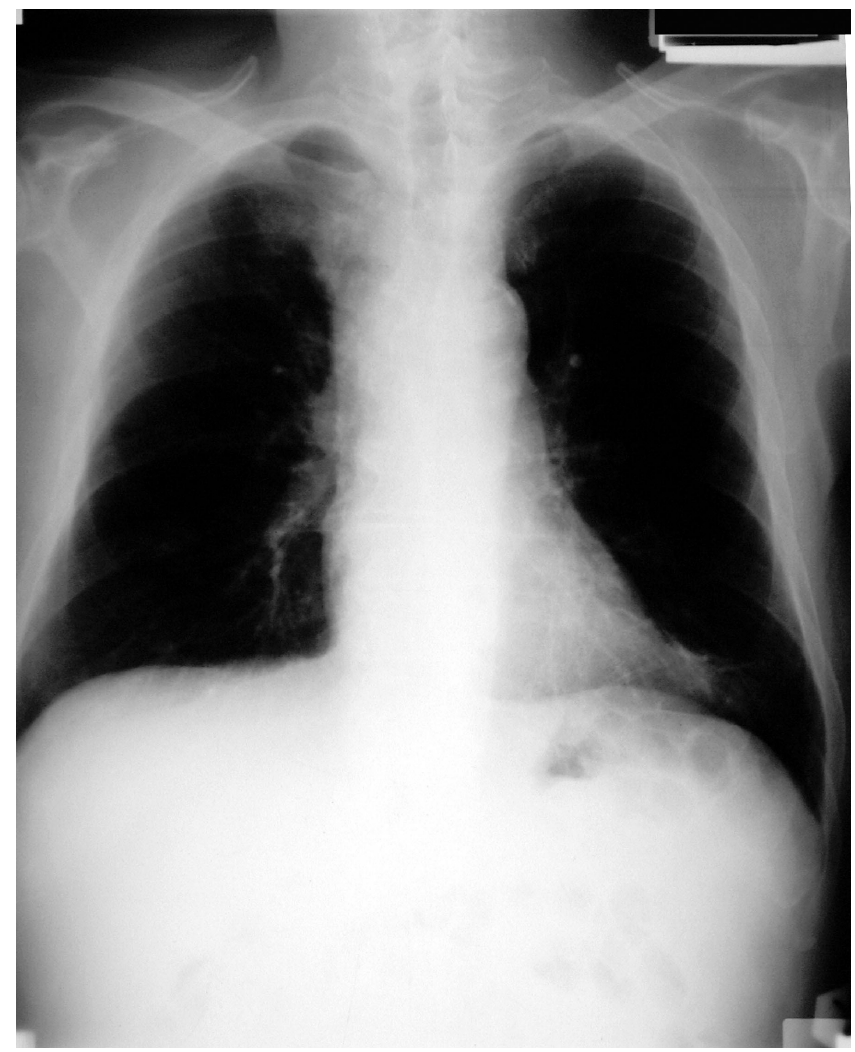

Figure 1. Chest radiograph of the patient with mediastinal enlargement caused by tuberculous lymphadenitis.

Antituberculosis treatment was administered to the patient. Most of the symptoms subsided after 1 month of treatment. Four months later, pathologic findings were improved on plain chest radiography, and a repeat $\mathrm{CT}$ scan of the chest showed that the mediastinal lymphadenopathy was completely resolved.

\section{Discussion}

SVCS has been considered a thoracic emergency attributed primarily to thoracic malignancies. Avoidance of mediastinoscopy and use of chemoradiation to alleviate the bothersome and deteriorating symptoms of the patient have been recommended in the past. Actually, mediastinoscopy is no longer an absolute contraindication in SVCS but rather a useful tool to obtain a reliable diagnosis.

Isolated hilar or paratracheal lymphadenopathy is a common sign of tuberculous infection in children and in immunocompro- 


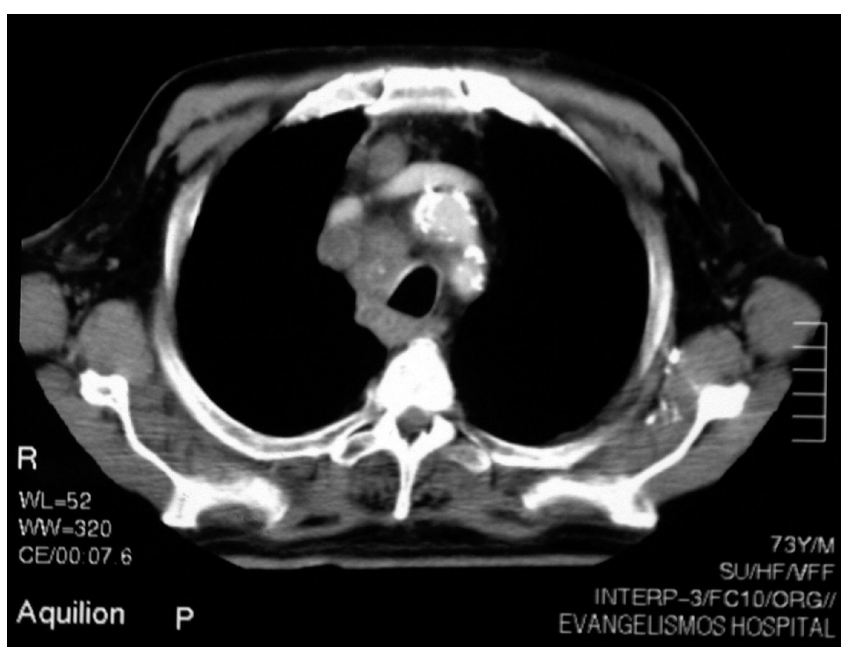

Figure 2. Thoracic computed tomographic scan demonstrating compression of the superior vena cava from the bulky lymph nodes occupying the space between the vena cava and the trachea.

mised adults with HIV infection and, if parenchymal lesions are absent, always presents a diagnostic problem. There is a low diagnostic yield from sputum examination and culture techniques, and therefore the patient is required to undergo procedures such as bronchoscopy or mediastinoscopy to establish the diagnosis.

Bronchoscopy usually is not very helpful and has a low diagnostic yield in isolated tuberculous mediastinal lymphadenopathy in the absence of lung lesions in contrast to the mediastinoscopy, which is effective in providing a tissue diagnosis with minimal morbidity and mortality.

Furthermore, a positive reaction to a tuberculin skin test or adenopathy with a low-density center and peripheral rim enhancement on a contrast-enhanced CT scan does not definitely confirm the diagnosis, and it has to be differentiated from diseases such as sarcoidosis, lymphoma, and metastases.

Some authors argue that transbronchial needle aspiration might solve this diagnostic problem, ${ }^{4}$ but in our case transbronchial needle aspiration was not helpful

Mediastinoscopy is the procedure of choice for the diagnosis of mediastinal masses or lymph nodes, provided that they are accessible. ${ }^{5}$ It provides easy access to the protracheal, paratracheal, and superior subcarinal lymph nodes. Numerous studies confirmed that mediastinoscopy is safe, and it has the advantage of direct visualization and palpation of the mediastinum, which allows larger biopsy specimens to be taken. In the presence of SVCS, the risk of hemorrhage exists with mediastinoscopy, but attention in the manipulations and cautious surgical hemostasis can prevent troublesome bleeding. The sensitivity, specificity, and accuracy of the method are between $94 \%$ and $100 \%$, and it causes minimal mortality and morbidity $(0.5 \%)$

In conclusion, tuberculosis can be the cause of lymph node disease of the mediastinum without any intraparenchymal lesions not only in children or immunocompromised adults with HIV infection and not only in developing countries but also in the so-called Western developed countries and in otherwise healthy adults and should always be included in the differential diagnosis. Mediastinoscopy is a reliable and accurate method to obtain the diagnosis and can be safely used in patients with SVCS.

\section{References}

1. Mclntire FT, Sykes EM Jr. Obstructive of the superior cava: a review of the literature and report of two personal cases. Ann Intern Med. 1949; 30:925-60.

2. Schechter MM. The superior vena cava syndrome. Am J Med Sci. 1954;227:46-56.

3. Nieto AF, Doty DR. Superior vena cava obstruction: clinical syndrome, etiology and treatment. Curr Prob Cancer. 1986;10:442-84.

4. Bilaceroglu S, Gunel O, Eris N, Cagirici U, Mehta AC. Transbronchia needle aspiration in diagnosing intrathoracic tuberculous lymphadenitis. Chest. 2004;126:259-67.

5. Ayed AK, Behbehani NA. Diagnosis and treatment of isolated tuberculous mediastinal lymphadenopathy in adults. Eur J Surg. 2001;167: $334-8$ 